\title{
Uncertainty quantification of modeling and simulation for reliability certification
}

\author{
Zhibo Ma \& Ming Yu \\ Institute of Applied Physics and Computational Mathematics, China
}

\begin{abstract}
When a systematic test is difficult to be implemented, M\&S (modeling and simulation) will be an important approach to get information on reliability certification, and the uncertainty of M\&S plays a key role in the certification framework such as QMU (quantification of margin and uncertainty). The model in $M \& S$ can be classified as an entity model and a physics model which represents the object of $\mathrm{M} \& \mathrm{~S}$ and the physical laws respectively. According to the mechanism that performances of a system are determined by initial conditions and the behavior of materials, the essential of M\&S can be depicted by a functional, which reveals how the total uncertainty of $M \& S$ is introduced from three sources such as entity modeling, physics modeling and computing. $\mathrm{M} \& \mathrm{~S}$ is on purpose to precisely forecast those un-happened physical processes but it must firstly go through V\&V (verification and validation) in which the uncertainty is quantified through comparison and the design variables of these entity models form a V\&V domain, then we think these uncertainties could be extrapolated to application domain in which the $M \& S$ is used as prediction and a no reference result is available for comparison. A hypothesis is brought forward that the total uncertainty varies along with the design variables of entity models, when physics modeling and the computing method remains unchanged, the relationship between uncertainty and variables of an entity model may be kept effectively, by which the uncertainty quantification (UQ) in application domain is reached. A series of detonation models are designed to demonstrate the above methods, the examples prove that the uncertainty in application domain could be well quantified unless the entity model in the application domain is far from the $\mathrm{V} \& \mathrm{~V}$ domain.
\end{abstract}

Keywords: uncertainty quantification, modeling and simulation, verification and validation, uncertainty prediction, reliability certification. 


\section{Introduction}

Reliability of physical systems is often evoked by two fundamentally different types of uncertainty, one is referred to as aleatory uncertainty due to inherent randomness in the systems including surroundings, environments, and scenarios, the other is referred to as epistemic uncertainty due to our lack of knowledge of the systems, and the laws about system operating, the epistemic uncertainty can farther be divided into recognized uncertainty and blind uncertainty [1]. In many cases, reliability certification is supported by experiment as it can quantify the aleatory uncertainties with high confidence and reduce the epistemic uncertainties efficiently. When experiment is prohibited, M\&S will be used to predict the performance of systems and turns into a great pillar to support reliability certification, but it is far from easiness for M\&S to take over the role that had ever been played by experiment [2-7]. Although computing results are finally offered by computers, M\&S is still a kind of subjective behavior as it is dominated by human being, so epistemic uncertainty is doomed to be its byproduct in addition to computing results. Consequently, quantification of this uncertainty will be necessary when $M \& S$ is greatly relied in reliability engineering.

When microstructure-defects of materials have little effect on system responses, such as in fluid or detonation devices, stochastic fatal failures can be ignored and the system can be considered as one-to-one relationship from design parameters to performance parameters, but the epistemic uncertainty about the relationship will be a great concern especially as the experiment can't be fulfilled. In this case, quantification of margin and the uncertainty of the quantified margin are all necessary, in which the margin are defined as the balance of the worst performance exceeding to the lowest demand and the uncertainty generally has the character of epistemic. This is the basic idea of QMU method, in which certification is done by the criterion that the confidence factor $\mathrm{C}=\mathrm{M} / \mathrm{U}$ is great than unity or not.

According to the requirements of different tasks, we have two kinds of M\&S characterized as deterministic and stochastic respectively. The former aims to find the deterministic relationship between the system parameters and the performance parameters, whereas the latter aims to find the stochastic relationship when the system and the operating process have randomness. As the simulation results of stochastic process may be mingled with uncertainties that originated from engineering systems, uncertainty of M\&S should be firstly referred to as deterministic M\&S and should be comprehended as deviation of simulation result to the true value. For stochastic process, the uncertainty of $M \& S$ should indicate the uncertainty when $M \& S$ is used to cognize the statistic law between the random inputs of system and the random performances, in which the statistic law is deterministic objectively. Generally, the UQ (uncertainty quantification) of deterministic $M \& S$ will offer a firm foundation for the UQ of stochastic M\&S.

In this paper, we are absorbed in deterministic M\&S and aim at UQ method considering $M \& S$ is applied on prediction. Firstly, we attempt to build a UQ 
principle of $M \& S$ for reliability certification, then the UQ method and its proceeding are illuminated, and an example is finally presented to demonstrate the ideas and methods of UQ.

\section{Principles of UQ of $M \& S$}

In traditional M\&S the UQ has not been thought highly enough as the test is the decisive factor in reliability certifications, some basic concepts about UQ have not obtained a profound comprehension and its promotion to the developing of M\&S has not been adequately realized. In order to standardize the practice of UQ, it is needed to draw a set of principles as follows:

(1) The premise that UQ can be done is that the technical state of M\&S must be relatively fixed and the computation can be fulfilled without the help of manual intervention;

(2) The final aim of UQ is to quantify the uncertainty of M\&S when it is used as expected application in which no reference solution is available for comparison;

(3) The uncertainty of reliability $M \& S$ should be apprehended as the deviation of simulation result to the true solution [8-13];

(4) The interval formed by the simulation result and uncertainty should cover the true solution;

(5) The UQ methods should be propitious to diagnose and eliminate the problems of M\&S so that the uncertainty can be effectively decreased, this leads to the demand to build the system hierarchy according to the coupling and complexity of phenomena;

(6) The UQ methods should be designed to have the favor to guarantee the adequacy of the input information and the creditability of UQ result.

\section{Configuration of $M \& S$ uncertainty}

For most engineering problems, the essence of $M \& S$ is to find the mapping relationship from the initial state and boundary condition to the system performance, which could be described as a functional:

$$
\zeta\left(t_{e}\right)=P\left[\xi\left(\boldsymbol{x}, t_{0}\right), \omega(\boldsymbol{x}, t), \eta(\xi),\left.t\right|_{t=t_{e}}\right.
$$

$\boldsymbol{x}$ : space coordinate of particles;

$t$ : variable of time, the subscripts " 0 " and "e" represent the "beginning" and "ending" respectively;

$\xi$ : parameters about physics state of particles;

$\omega$ : functions about boundary conditions;

$\eta$ : functions of $\xi$ to describe the behavior laws of matters in the system;

$\zeta$ : system performances which vary with time and other functions;

$P$ : the functional to show the general laws of physics. 
At the mention of modeling of M\&S, we will have two kind of models, one we call entity model corresponding the function $\xi\left(\boldsymbol{x}, t_{0}\right)$ and $\omega(\boldsymbol{x}, t)$, the other we call physics model corresponding the function $\eta(\xi)$ and the functional $P[\cdot]$, the final aims of uncertainty quantification of $M \& S$ should refer to the uncertainty of numerical results when the $M \& S$ is applied at new entity models that have no reference solutions.

According to equation (1), we can partition the uncertainty of M\&S into three parts as:

$U_{1}$ : M\&S uncertainty due to the entity modeling uncertainty $U_{1}^{0}$ when building the $\xi\left(\boldsymbol{x}, t_{0}\right)$ and $\omega(\boldsymbol{x}, t)$

$U_{2}$ : M\&S uncertainty due to the physics modeling uncertainty $U_{2}^{0}$ when building the $\eta(\xi)$ and $P[\cdot]$

$U_{3}$ : M\&S uncertainty due to numerical computation when resolving the functional $P[\cdot]$

So the total uncertainty of M\&S may be presented as

$$
U_{M \& S}=U_{1} \oplus U_{2} \oplus U_{3}
$$

where " $\oplus$ " means the total uncertainty is consist of three parts, in some special cases, it may approximately equal to the summation of the three parts.

For the modeling induced uncertainty $U_{1}$ and $U_{2}$, the modeling uncertainties of $U_{1}^{0}$ and $U_{2}^{0}$ must be firstly quantified, then through transference to we can get $U_{1}$ and $U_{2} \cdot U_{1}^{0}$ and $U_{2}^{0}$ both has two parts, one is the model form uncertainty $U_{\text {form }}$ and the other is the model parameter uncertainty $U_{\text {para }}$, the uncertainty $U_{3}$ is mainly induced by numerical discretization, numerical iteration and number round up etc.

\section{Main techniques needed in UQ of M\&S}

In order to successfully quantify the uncertainty of M\&S, some pillars of techniques should be built up as follows:

(1) Building the validation tier of engineering system

In order to easily segregate and diagnose the problems and collect the uncertainty information of $\mathrm{M} \& \mathrm{~S}$, a multilayer hierarchy for a complex system must be built according to the system operating process with time or according to the coupling extent of different phenomena.

(2) Uncertainty quantification of modeling

UQ of modeling aims at quantifying the $U_{1}^{0}$ and $U_{2}^{0}$. To different models, there are corresponding techniques in scientific theory, measurement and experiment statistics which are used to meet this demand. 
(3) Transfer analysis of uncertainty

Transfer analysis is used to quantify the $U_{1}$ and $U_{2}$ which are induced by $U_{1}^{0}$ and $U_{2}^{0}$, it would be implemented with the help of computing program, and be denoted by variations as

$$
\begin{gathered}
U_{1}=\left|\delta P\left[\xi\left(\boldsymbol{x}, t_{0}\right), \omega(\boldsymbol{x}, t), \eta(\xi), t\right]\right|=\left|L\left[\xi\left(\boldsymbol{x}, t_{0}\right), \omega(\boldsymbol{x}, t), \eta(\xi), t, \delta \xi\left(\boldsymbol{x}, t_{0}\right), \delta \omega(\boldsymbol{x}, t)\right]\right| \\
U_{2}=\left|\delta P\left[\xi\left(\boldsymbol{x}, t_{0}\right), \omega(\boldsymbol{x}, t), \eta(\xi), t\right]\right|=\mid L\left[\xi\left(\boldsymbol{x}, t_{0}\right), \omega(\boldsymbol{x}, t), \eta(\xi), t, \delta \eta(\xi), \delta P[\cdot] \mid\right]
\end{gathered}
$$

(4) Comparison between simulation and reference solutions

Based on V\&V techniques, uncertainties of $M \& S$ are quantified by comparison with the high confidence reference solutions, the design of benchmark entity model and construction of its reference solution is the important support of $\mathrm{V} \& \mathrm{~V}$.

(5) Extrapolation of uncertainty

The actual value of M\&S lies on the engineering prediction for new entity model, for which the uncertainty cannot be quantified by comparison, in this case, uncertainty should be inferred according to the law that uncertainty varies with the parameters of entity models, this law can be presented by a polynomial function and can generally be determined by the comparison information of the entity models which have reference solutions.

(6) Synthesis of uncertainty information from different types

The M\&S on a new system often has more than one source of uncertainties, such as the uncertainty of performance transferred from modeling uncertainty, the uncertainty transferred from the M\&S of lower tier and that extrapolated from same tier of system, in addition, the comparison between different software may produce another information of M\&S uncertainty. This information could be synthesized by generalized information theories.

\section{Uncertainty extrapolation to application domain}

In $\mathrm{V} \& \mathrm{~V}$ activities, the design parameters of entity model, including the environment parameters will form a multi-dimensional space and it can be divided into validation domain and application domain. Validation domain is the space that validation experiments have occupied, and the application domain is the space that the M\&S is expected to be applied. As there is no experiment data used to compare, its uncertainty should be inferred according to the uncertainty in validation domain and the relationship of uncertainties between these two domains.

In order to reveal the relationship of the uncertainties between the two domains, we offer a series of hypothesis as follows:

(1) If the instrument and method remain unchanged, the uncertainty of measurement on physics parameters such as size, mass, density, temperature and velocity etc., is a function of the measurand; 
(2) If a dependent variable is educed from some measurands with definite methods, then the uncertainty of the dependent variable is a function of the misbrands;

(3) If the computing program is solidified, the uncertainty aroused by numerical computation is a function of the design parameters of entity model;

(4) Based above assumptions, the total uncertainty of M\&S could be presented as a function of design parameters of entity model:

$$
U_{M \& S}=U_{1}[\cdot] \oplus U_{2}[\cdot] \oplus U_{3}[\cdot]=U\left[\xi\left(\boldsymbol{x}, t_{0}\right), \omega\left(\boldsymbol{x}, t_{0}\right)\right]
$$

(5) Above function is simultaneously fit for validation and application domains.

According to these five assumptions, if the function in (5) is determined within the validation domain, the uncertainty in application domain may be quantified by extrapolations.

\section{Steps of UQ about M\&S}

According to the principle of UQ and the ideas of V\&V, the steps of UQ are recommended here:

(1) Following the need in reliability certification, the demand about the magnitude of M\&S uncertainty is ascertained;

(2) To build the validation tiers of system that the M\&S will be applied on;

(3) To build the benchmark models and their credible reference solutions;

(4) To develop code verification based on the benchmark models, and assess the uncertainty that induced by numerical computation by comparison, also the bugs of program is detected and the convergence and stability of algorithm are verified;

(5) To develop solution verification and thus the accuracy of computation is evaluated, generally no reference solution can be used to compare in solution verification, GCI (Grid Convergence Index) is used to assure the true value is covered by the interval formed by simulation result and its uncertainty;

(6) Aim at expected application of $M \& S$, design and develop calibration experiments of each system tier, the parameters of computation and physics model, including knobs are adjusted and then fixed;

(7) To execute validation experiment and M\&S of each system tier, calibrated parameters are used in $M \& S$ and the uncertainty is quantified by comparison with the experiment data;

(8) To extrapolate the uncertainty from validation to application domains;

(9) To transfer the uncertainty of modeling to the M\&S results of system performance and thus obtain uncertainty that induced by entity modeling and physics modeling;

(10) To synthesize the uncertainties from extrapolation and transference, reach the aim to quantify the total uncertainty of $M \& S$ corresponding to expected application. 


\section{Example of UQ about M\&S}

\subsection{Entity model}

The entity model contains a detonator and a block of explosive, the thickness of explosive at the symmetric axis is $\delta$, this model and the experiment facility are used to investigate the initial process of the detonation developed after the explosive is ignited, from which the physics model of ignition and the spread of detonation wave are built. The data to be measured in this test is the peak velocity of the explosive particle close to the $\mathrm{LiF}$ window at the axis when detonation wave reach the right side of explosive, the measuring error of this velocity is $\pm 3.0 \%$, as shown in Fig. 1 .

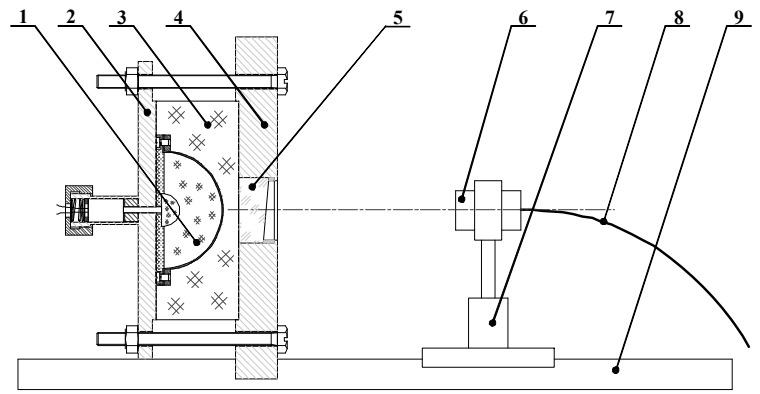

1-Detonator;

2-Upper fixing plate;

3-Main explosive;

4-Window fixing plate;

5-LiF window;

6-Laser detector;

7-Bracket of detector;

8-Optical fiber;

9-Reference plate.

Figure 1: The entity model and test facility.

\subsection{System tier}

This entity model is regarded as a system and be divided into two tiers:

(1) Detonator tier: only contains detonator, explosive is excluded;

(2) System tier: contains detonator and explosive simultaneously.

The detonation tier must experience the calibration and validation firstly to assess and build the credibility of M\&S when simulating the inner operating process of detonator, the calibration, validation and application of $M \& S$ on the system tier should be implemented whereafter.

\subsection{Model calibration}

Model calibration must be done before model validation to adjust and fix the physics models, which include the model forms and model parameters.

The calibration is divided into two steps:

(1) Calibration at detonator tier

The calibration metric of this tier is the peak velocity of the half sphere metal film in the center position of the detonator, by comparison of the simulation results and test data of the peak velocities, the physics model and computing parameters of the detonator are adjusted and fixed. 
(2) Calibration at system tier

The calibration metric of this tier is the peak velocity of the explosive close to the $\mathrm{LiF}$ window, different thickness of explosive is selected to form different entity models, through comparison between the simulation results and test data, parameters of physics model and computation are adjusted and fixed, such as the spread velocity of detonation wave, the parameters of JWL equation of state of the reaction product of the main explosive.

\subsection{Model validation}

In this entity model, the explosive thickness $\delta$ is the only design variable, the density of the explosive is not regarded as design variable. What we concern in this problem is how the M\&S uncertainty of the main explosive peak velocity $V$ varies with the thickness $\delta$. When manufacturing the entity models, the thickness and density of the explosive will have randomness, so their real values should be measured individually when M\&S is executed on these entity models.

Validation experiments are divided into 4 groups, for which the $\delta$ is designed as $5 \mathrm{~mm}, 10 \mathrm{~mm}, 15 \mathrm{~mm}$ and $20 \mathrm{~mm}$ respectively. In each group 2 entity models are manufactured and both of them have the same designed entity parameters. Owing to the randomness of manufacture, the thickness and density of each explosive sample may have some deviations compare to the designed values in the range of tolerances.

In validation activity, the parameters of physics model such as in JWL equation of state keep unchanged as calibration has been finished, then we have M\&S results about the peak velocity $V$ of the 8 entity models, as in table 1 , and the corresponding test data is listed in table 2.

Here we define the simulation error as the difference of the simulation result and test data:

$$
E_{V}=V^{\text {simulation }}-V^{\text {test }}
$$

Each group has 2 test data, we pick up the uncertainties of M\&S from the larger absolute values of the two errors, as in table 3 .

Table 1: Simulation results of $V$ in the validation domain $\left(\delta / m m, V / m \cdot \mathrm{s}^{-1}\right)$.

\begin{tabular}{lcccc}
\hline & $\delta=5.0$ & $\delta=10.0$ & $\delta=15.0$ & $\delta=20.0$ \\
\hline Simulate (1) & 2020.97 & 2042.91 & 2118.81 & 2170.32 \\
Simulate (2) & 2012.26 & 2043.21 & 2137.94 & 2196.56 \\
\hline
\end{tabular}

Table 2: $\quad$ Test results of $V$ in the validation domain $\left(\delta / m m, V / m \cdot \mathrm{s}^{-1}\right)$.

\begin{tabular}{lcccc}
\hline & $\delta=5.0$ & $\delta=10.0$ & $\delta=15.0$ & $\delta=20.0$ \\
\hline Test (1) & 2082.18 & 2092.99 & 2110.74 & 2129.19 \\
Test (2) & 1946.33 & 1993.91 & 2109.14 & 2148.46 \\
\hline
\end{tabular}


Table 3: Simulation errors and uncertainties of $V$ in the validation domain $\left(\delta / m m, E_{V} / m \cdot \mathrm{s}^{-1}, U_{V} / m \cdot \mathrm{s}^{-1}\right)$.

\begin{tabular}{lcccc}
\hline & $\delta=5.0$ & $\delta=10.0$ & $\delta=15.0$ & $\delta=20.0$ \\
\hline EV(1) & -61.21 & -50.08 & 8.07 & 41.13 \\
EV(2) & 74.93 & 49.30 & 28.80 & 48.10 \\
UV & 75.0 & 50.0 & 30.0 & 50.0 \\
\hline
\end{tabular}

\subsection{Uncertainty quantification in application domain}

The design range of explosive thickness $\delta$ in validation domain is $5 \mathrm{~mm} \sim 20 \mathrm{~mm}$, here we define the entity models in application domain which have the $\delta$ of $2 \mathrm{~mm}, 3 \mathrm{~mm}, 4 \mathrm{~mm}$ and $25 \mathrm{~mm}$, it is obvious that the two domains do not overlap.

In practice, there is no test data used to compare with the simulation results, so it is necessary to build a prediction model for quantifying the uncertainty in application domain.

Based on the idea of expression (5), we use a second-order polynomial to construct the prediction model of uncertainty, from which the law of uncertainty varies with $\delta$ is extracted:

$$
U(\delta)=a_{0}+a_{1} \delta+a_{2} \delta^{2}
$$

With the data in table 3 , we have

$$
\left(\begin{array}{ccc}
1 & 5 & 25 \\
1 & 10 & 100 \\
1 & 15 & 225 \\
1 & 20 & 400
\end{array}\right)\left(\begin{array}{l}
a_{0} \\
a_{1} \\
a_{2}
\end{array}\right)=\left(\begin{array}{l}
75 \\
50 \\
30 \\
50
\end{array}\right)
$$

Equation (8) is generally super posed, based on the principle of minimum 2ndnorm solution, we have

$$
\left(\begin{array}{lll}
a_{0} & a_{1} & a_{2}
\end{array}\right)^{T}=\left(\begin{array}{lll}
131.25 & -13.15 & 0.45
\end{array}\right)^{T}
$$

Up to now, expression (7) has been fully determined, from which the uncertainties in application domain are quantified, as in table 4.

Table 4: $\quad$ Predicted M\&S uncertainties of $V$ in the application domain $\left(\delta / m m, U_{V} / m \cdot \mathrm{s}^{-1}\right)$.

\begin{tabular}{ccccc}
\hline & $\delta=2.0$ & $\delta=3.0$ & $\delta=4.0$ & $\delta=25.0$ \\
\hline Predicted-U $_{\mathrm{V}}$ & 106.75 & 95.85 & 85.85 & 83.75 \\
\hline
\end{tabular}




\section{Checkout of the UQ method}

In this paper, we only attempt to check the extrapolation-based UQ method for application domain.

Based on above entity models, checkout is executed through following steps:

(1) According to the expression (7), extrapolate the M\&S uncertainties to application domain as in table 4;

(2) For each $\delta$ in application domain, to manufacture 2 entity models, and draw up a test plan for the 8 entity models in all;

(3) To measure the real $\delta$ and real explosive density of the 8 entity models, then execute $M \& S$ and freeze up the numerical results of the 8 models;

(4) To implement tests for the 8 models and record the test data;

(5) To subtract test data from simulation results, and get 8 simulation errors;

(6) To check the accuracy of UQ in application domain by comparing the simulation errors in table 5 and the extrapolated uncertainties in table 4 .

Table 5: Simulation or test results and errors of $V$ in the application domain $\left(\delta / m m, V / m \cdot \mathrm{s}^{-1}\right)$.

\begin{tabular}{lcccc}
\hline & $\delta=2.0$ & $\delta=3.0$ & $\delta=4.0$ & $\delta=25.0$ \\
\hline Simulate (1) & 1889.76 & 1946.91 & 2011.37 & 2199.69 \\
Simulate (2) & 1895.68 & 1944.80 & 2012.45 & 2202.41 \\
Test (1) & 1781.99 & 1850.83 & 2062.45 & 2179.22 \\
Test (2) & 1902.39 & 1935.41 & 1928.27 & 2114.38 \\
Error (1) & $\mathbf{1 0 7 . 7 7}$ & $\mathbf{9 6 . 0 8}$ & -51.08 & 20.47 \\
Error (2) & -6.71 & 9.39 & $\mathbf{8 4 . 1 8}$ & $\mathbf{8 8 . 0 3}$ \\
\hline
\end{tabular}

From table 5, when paying more attention to the error that has a larger absolute value in each $\delta$, we will find that the extrapolation can basically reflect the law that M\&S uncertainty varies with the entity variable $\delta$ and consequently has the prediction ability. As the test data are not adequacy, the UQ in application domain has not yet strictly meet the principle about true value covering, and with the entity model getting farther away from the validation domain, the accuracy of the extrapolation will get decreased.

\section{Conclusion}

M\&S will become more and more important in reliability engineering and the effective quantification of its uncertainty can markedly decrease the misplay of decision making. As the ultimate goal of $M \& S$ is being applied to new entity models, a series of hypothesis have been offered to support the idea that the uncertainty of M\&S could be presented as a function of entity parameters, from which the uncertainty extrapolation from validation domain to application domain has been built. Two examples about one dimensional Riemann problem in reference [14] and two-dimensional detonation model in this paper have shown the effectiveness of this method, but if the extrapolation is used on the model too far away from the validation domain, the accuracy of UQ in 
application domain will decrease especially when blind uncertainty exists in the M\&S.

\section{References}

[1] Oberkampf W L, Roy C J. Verification and validation in science computing [M], Cambridge University Press, 2010, 1-767.

[2] Ma Zhibo, Ying Yangjun, Zhu Jianshi. QMU certifying method and its implementation $[\mathrm{J}]$. Chinese Journal of Nuclear Science and Engineering, 2009, 29 (1): 1-9.

[3] Pilch M, Trucano T G, Helton J C. Ideas underlying the quantification of margins and uncertainties [J]. Reliability Engineering and System Safety, 2011, 96: 965-975.

[4] Helton J C. Quantification of margins and uncertainties: Conceptual and Computational Basis [J]. Reliability Engineering and System Safety, 2011, 96: 976-1013.

[5] Doostan A, Iaccarino G. A least-squares approximation of partial differential equations with high-dimensional random inputs [J]. Journal of Computational Physics, 2009, 228: 4332-4342.

[6] Helton J C, Johnson J D, Sallaberry C J, Storlie C B. Survey of samplingbased method for uncertainty and sensitivity analysis [J]. Reliability Engineering and System Safety, 2006, 91(10-11): 1175-1209.

[7] Roy C J, Oberkampf W L. A comprehensive framework for verification, validation, and uncertainty quantification in scientific computing [J]. Computer methods in applied mechanics and engineering, 2011, 200: 2131-2144.

[8] American Institute of Aeronautics and Astronautics, Reston, VA, Guide for Verification and Validation of Computational Fluid Dynamics [S]. AIAA G-077-1998.

[9] Ye Depei, Song Zhenguo, Wang Xianzhi, Expression and evaluation of uncertainty in measurement [S]. Military Standard of the People's Republic of China, 1999, GJB3756-99.

[10] The American Society of Mechanical Engineers, Test uncertainty [S], American National Standard, 2005, ASME PTC 19.1-2005.

[11] The American Society of Mechanical Engineers, Guide for verification and validation in computational solid mechanics [S], American National Standard, 2006, ASME V\&V 10-2006.

[12] The American Society of Mechanical Engineers, Standard for verification and validation in computational fluid dynamics and heat transfer [S], American National Standard, 2009, ASME V\&V 20-2009.

[13] The American Society of Mechanical Engineers, An illustration of the concepts of verification and validation in computational solid mechanics [S], American National Standard, 2012, ASME V\&V 10.1-2012.

[14] Ma Zhibo, Zheng Miao, Yin Janwei, et al. Quantification of uncertainties in detonation simulations [J]. Chinese Journal of Computational Physics, 2011, 28 (1): 66-74. 